\title{
Estudo comparativo dos diagnósticos macro e microscópicos de autópsias: análise de dificuldades e possiveis causas de erro
}

Primeira submissão em 09/05/03 Última submissão em 09/12/03 Aceito para publicação em 26/01/04 Publicado em 20/10/04

\section{Comparative study of gross and microscopic diagnosis of autopsies: analysis of difficulties and eventual error causes}

Camilo de Viterbo Idalino'; Eliane Espindola Gomes²; Patricia Maluf Cury ${ }^{3}$

\begin{tabular}{l|l}
\multicolumn{1}{c|}{ Unitermos } & resumo \\
Atestado de óbito & $\begin{array}{l}\text { Introdução: O diagnóstico do atestado de óbito é muito utilizado em estatísticas de saúde pública, } \\
\text { entretanto o seu preenchimento se baseia apenas no diagnóstico macroscópico da autópsia. Quando } \\
\text { comparamos a macroscopia com o laudo definitivo da autópsia (microscopia), percebemos que a micro } \\
\text { Microscopia }\end{array}$ \\
étópsia & $\begin{array}{l}\text { é decisiva, muitas vezes modificando o laudo do atestado. Objetivos: Avaliar prospectivamente as dife- } \\
\text { renças entre os diagnósticos macro e microscópico de causa de óbito e as limitações do atestado como } \\
\text { ferramenta epidemiológica. Metodologia: Estudamos } 100 \text { autópsias realizadas pelo Departamento de } \\
\text { Patologia da Faculdade de Medicina de São José do Rio Preto (FAMERP) entre julho de } 2000 \text { e maio de } \\
\text { 2001, sendo } 65 \text { homens e } 35 \text { mulheres com idade média de } 44,17 \text { anos. As causas básicas e imediatas } \\
\text { do óbito eram obtidas do atestado e comparadas ao diagnóstico do laudo definitivo de cada autópsia. } \\
\text { Resultados: Das cem autópsias, 16 tiveram o diagnóstico de causa imediata discordante e 22, discordância } \\
\text { na causa básica. As divergências foram principalmente em pulmões, rins, fígado e coração. Conclusões: } \\
\text { O atestado de óbito, embora muito utilizado, apresenta limitações como ferramenta epidemiológica, } \\
\text { e o estudo microscópico é decisivo, principalmente quando envolve os órgãos referidos, nos quais a } \\
\text { macroscopia apresenta uma limitação diagnóstica. }\end{array}$
\end{tabular}

abstract

Background: The diagnosis of death certificate is very used in statistics of public health. However, its completion is only based on the macroscopic diagnosis of autopsy. When comparing macroscopia with the autopsy's final award, we realize that microscopia is decisive and modifies death certificate's award many times. Objectives: Prospectively evaluate the differences between gross and microscopic disgnosis of death cause and the limitations of death certificate as an epidemiologic tool. Method: 100 autopsies were performed and studied at the Departamento de Pathologia da Faculdade de Medicina de São José do Rio Preto, between July, 2000 and May, 2001. A total of 65 men and 35 women were studied and they were 44,17 years old on average. The basic and the immediate causes of obit were taken from death certificate and compared with the diagnosis on the final award of each autopsy. Results: Based on the total of 100 autopsies, 16 had a discordant diagnosis on the immediate cause and 22 discordant cases on the basic cause of death. Discordance were mainly on lungs, kidneys, liver and heart. Conclusions: Although death certificate is very used, it shows limitations as an epidemiologic tool. As a consequence, the microscopic study is conclusive especially when it is related to the mentioned organs, in which macroscopia shows a diagnostics limitation. key words

Death certificate

Macroscopia

Microscopia

Autopsy

1. Aluno do sexto ano de medicina da Faculdade de Medicina de São José do Rio Preto (FAMERP), com bolsa de iniciação científica da Fundação de Amparo à Pesquisa do Estado de São Paulo (FAPESP).

2. Residente de Patologia do Departamento de Patologia da FAMERP.

3. Médica-patologista; professora-doutora do Departamento de Patologia da FAMERP.

Trabalho realizado com auxilio de bolsa de iniciação científica da FAPESP (00/06625-2) e apresentado no XXIII Congresso de Patologia em 2001, Salvador, Bahia. 


\section{Introdução}

A autópsia é um procedimento extremamente importante para esclarecimento da causa do óbito e de patologias associadas. Além de confirmar ou alterar o diagnóstico clínico, muitas vezes ela também revela outros achados não-diagnosticados clinicamente ${ }^{(1)}$. Muitos estudos têm sido realizados com o intuito de comparar o diagnóstico clínico com o diagnóstico da autópsia, mostrando uma diferença de $11 \%$ a $60 \%$ entre os dois métodos $^{(2,4,5)}$.

Veress et al. analisaram a concordância entre vários patologistas em relação aos diagnósticos macroscópicos de autópsia. A conformidade foi muito boa em relação ao diagnóstico da causa básica do óbito; porém, quando avaliadas as doenças secundárias, a divergência foi maior, mostrando a necessidade de discussão entre os patologistas nos casos de diagnóstico macroscópico mais difícil. Em relação a estudos comparativos entre os diagnósticos macroscópico e microscópico de uma mesma autópsia, existem poucos trabalhos na literatura ${ }^{(3)}$. Esse tipo de estudo é interessante na medida em que as dificuldades inerentes à observação apenas macroscópica são mais bem avaliadas, além de ser um controle interno dos custos e da qualidade do patologista e do serviço.

Após a realização da necropsia, o patologista elabora um laudo preliminar com a macroscopia. Posteriormente, com as informações clínicas, macroscópicas e microscópicas, ele realiza um laudo definitivo. Este tem melhor acurácia que o atestado de óbito, pois está complementado pelos dados microscópicos. Infelizmente, o atestado de óbito é um documento oficial que não pode ser mudado e, portanto, o laudo definitivo é um documento arquivado no hospital e que dificilmente entra nos estudos epidemiológicos. Estes são geralmente baseados nos atestados de óbito, os quais, muitas vezes, não condizem com a realidade. $O$ conhecimento das dificuldades inerentes à macroscopia é de importância não somente para o próprio patologista, como para a interpretação dos estudos baseados apenas nos diagnósticos fornecidos pelo atestado de óbito.

Dessa forma, este trabalho visa comparar o diagnóstico macroscópico ao microscópico de uma mesma autópsia, com o intuito de verificar as diferenças entre os dois métodos e tentar avaliar quais as dificuldades diagnósticas inerentes à limitação da macroscopia, e aquelas secundárias a erros de diagnóstico que eventualmente poderiam ser evitados.

\section{Material e método}

Foram analisadas cem autópsias consecutivas, excluindo-se os natimortos, realizadas no Departamento de Patologia da Faculdade de Medicina de São José do Rio Preto (FAMERP), durante o período de julho/2000 a maio/2001. O diagnóstico macroscópico foi baseado nos dados presentes no atestado de óbito de cada autópsia, juntamente com um documento interno de diagnósticos macroscópicos realizado no momento da autópsia. Desses documentos, as informações do paciente foram coletadas e ordenadas da seguinte forma: sexo, idade, causa básica de morte, causas conseqüenciais, causa final, causas contributivas e outras doenças. O diagnóstico microscópico foi obtido pelos laudos definitivos de cada autópsia, fornecidos pelo patologista após o estudo de lâminas histológicas. Do laudo definitivo obtivemos os dados de causa final da morte, causa básica e outras doenças. Após a obtenção dos mesmos, realizamos o estudo comparativo dos diagnósticos macro e microscópicos das autópsias, buscando a concordância e a discordância entre os dados.

\section{Resultados}

Dos pacientes submetidos à necropsia, 65 eram homens e a média de idade de todo o grupo foi de 44,2 anos (de três meses a 86 anos). Em relação às causas básicas de morte, tendo como fonte os dados contidos no atestado de óbito (AO), ou seja, baseado em dados macroscópicos, notamos um predomínio das doenças cardiovasculares, representando $35 \%$ dos casos, seguidas pelas doenças do trato digestório, em $15 \%$ dos casos. Quando avaliamos as causas terminais de morte descritas no AO, observamos que as doenças respiratórias responderam por $34 \%$ dos casos, seguidas por doenças cardiovasculares, representando $24 \%$.

Ao analisar a discordância entre as causas imediatas de morte, comparando macro e microscopia, percebemos que 16 casos foram discordantes. Destes, oito casos divergiram em relação às doenças do aparelho respiratório e, em cinco casos, às cardiovasculares. Considerando apenas as causas básicas de morte, a discordância ocorreu em 22 necropsias, sendo que as principais divergências aconteceram nas doenças cardiovasculares (dez casos), seguidas das doenças do aparelho respiratório (quatro casos). Em relação aos diagnósticos macroscópicos não-confirmados pela microscopia, houve prevalência das doenças do aparelho respiratório (21 casos discordantes), seguidas pelas doenças renais e 
hepáticas, discordantes em 19 casos. Já as doenças cardiovasculares não foram confirmadas microscopicamente em dez casos (Figura 1).

Ao analisarmos e compararmos os diagnósticos microscópicos que não foram evidenciados apenas com a análise macroscópica das autópsias, observamos os seguintes órgãos em que a discordância foi mais prevalente: pulmão (72 casos), rim (45 casos), fígado (45 casos) e coração (26 casos) (Figura 2).

Estudando especificamente os pulmões, as principais doenças não-confirmadas pela microscopia foram: edema agudo de pulmão (nove casos), broncopneumonia (quatro casos), insuficiência respiratória (três casos), hemorragia e hipertensão (dois casos cada). Nos rins, as doenças mais discordantes (nas quais a macroscopia não foi confirmada pela micro) foram: necrose tubular aguda (sete casos) e necrose arteriolar benigna (cinco casos). No fígado, a esteatose e a hepatopatia a esclarecer foram as mais prevalentes, com seis casos cada. Referente ao coração, a doença mais diagnosticada macroscopicamente e não-confirmada pela microscopia foi o infarto agudo do miocárdio (IAM) (cinco casos) (Tabela 1). A Tabela 2 descreve os principais diagnósticos microscópicos discordantes em relação a cada diagnóstico macroscópico.

Em relação aos pulmões, órgãos de maior discrepância diagnóstica macro e microscópica, observamos que as principais afecções pulmonares detectadas apenas na microscopia foram: broncopneumonia (12 casos), enfisema (dez casos) e hemorragia (nove casos). Nos rins, pielonefrite (19 casos), nefrosclerose arteriolar benigna (14 casos) e necrose tubular aguda (oito casos) foram os principais diagnósticos microscópicos não-evidenciados na macroscopia. No fígado, a maior discordância é representada pela esteatose (12 casos) e pela hepatite aguda (sete casos) e, em relação ao coração, o infarto antigo (nove casos) (Tabela 3). A Tabela 4 descreve os principais diagnósticos macroscópicos discordantes em relação a cada diagnóstico microscópico.

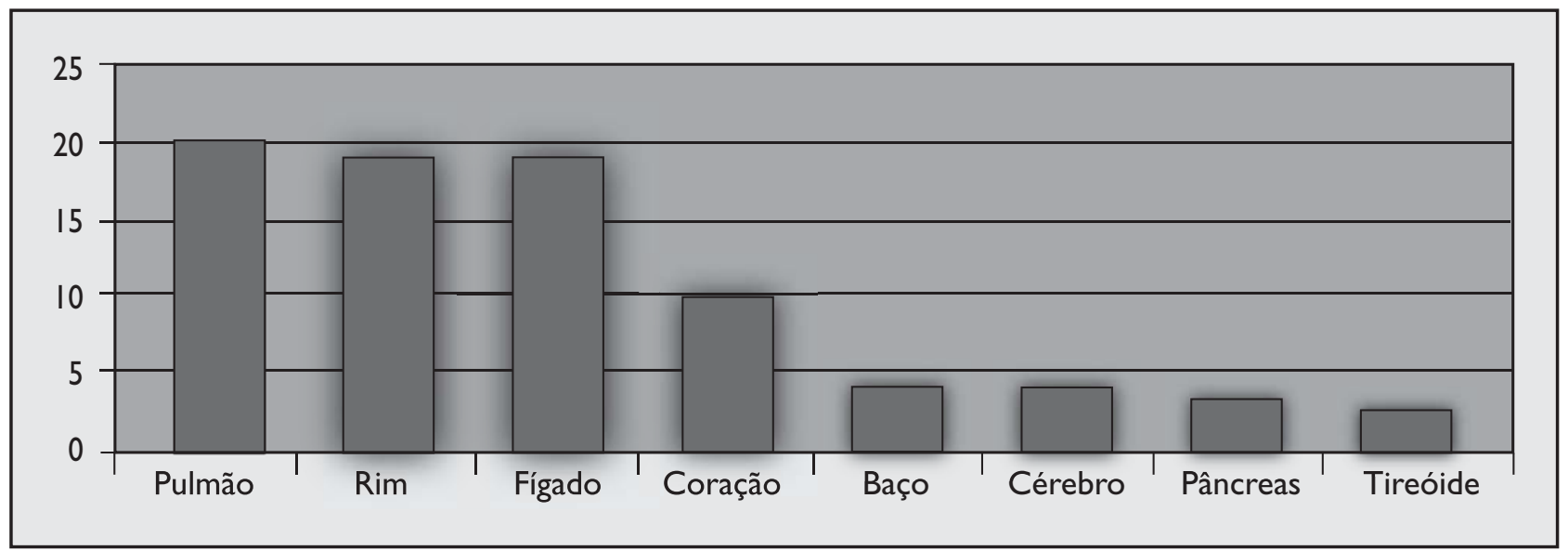

Figura 1 - Diagnósticos macroscópicos não-confirmados pela microscopia

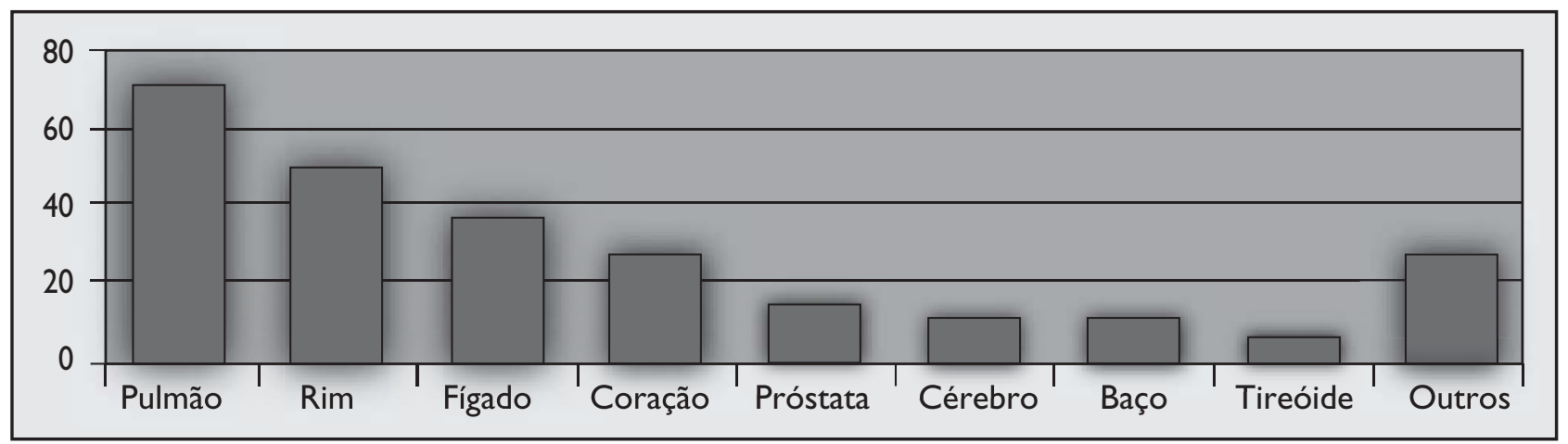

Figura 2 - Diagnósticos microscópicos não-evidenciados à macroscopia 
Tabela 1 Diagnósticos macroscópicos que não foram confirmados pela microscopia

\begin{tabular}{|l|c|l|l|l|l|l|c|}
\hline \multicolumn{1}{|c|}{ Pulmão } & \multicolumn{2}{c|}{ Rim } & \multicolumn{2}{c|}{ Coraçãão } \\
\hline $\begin{array}{l}\text { Edema agudo } \\
\text { Broncopneumonia }\end{array}$ & 9 & Necrose tubular aguda & 7 & Esteatose & 6 & Infarto agudo & 5 \\
\hline $\begin{array}{l}\text { Insuficiência } \\
\text { respiratória }\end{array}$ & 3 & $\begin{array}{l}\text { Pielonefrite } \\
\text { crônica }\end{array}$ & 4 & Hepatopatia & 6 & Chagas & 4 \\
\hline Hipertensão & 2 & Outros & & Hepatite & 3 & Cardiomegalia & 1 \\
\hline Hemorragia & 2 & - & 3 & Fibrose & 2 & Infarto antigo & 1 \\
\hline Outros & 5 & - & - & Outros & 2 & Aterosclerose & 1 \\
\hline Total & 25 & Total & - & - & - & - & - \\
\hline
\end{tabular}

Tabela 2 Diagnósticos macroscópicos divergentes e seus respectivos achados microscópicos

\section{a) Pulmão}

\begin{tabular}{l|l|l|c}
\hline Edema agudo (macro) & Broncopneumonia (macro) & 2 \\
\hline Normalidade & 3 & Normalidade & 1 \\
Tromboembolismo & 2 & Membrana hialina & 1 \\
Hemorragia & 2 & Dano alveolar difuso & - \\
Enfisema & 1 & - & - \\
Congestão & 1 & - & 4 \\
Total & $\mathbf{9}$ & Total & 4 \\
\hline
\end{tabular}

b) $\operatorname{Rim}$

\begin{tabular}{l|l|l|c}
\hline \multicolumn{2}{l|}{ Necrose tubular aguda (macro) } & Nefrosclerose (macro) & 3 \\
\hline Normalidade & 4 & Pielonefrite crônica & 2 \\
Pielonefrite crônica & 2 & Normalidade & - \\
Hemorragia & 1 & - & 5 \\
Total & 7 & Total & 5 \\
\hline
\end{tabular}

\section{c) Fígado}

\begin{tabular}{l|l|l|c}
\hline \multicolumn{2}{l|}{ Esteatose (macro) } & Hepatopatia (macro) & 3 \\
\hline Normalidade & 3 & Hepatite aguda & 2 \\
Congestão & 2 & Normalidade & 1 \\
Hepatite crônica & 1 & Congestão & 6 \\
Total & $\mathbf{6}$ & Total & \\
\hline
\end{tabular}

d) Coração

\begin{tabular}{l|l|l|c}
\hline Infarto agudo (macro) & Cardiopatia chagásica (macro) & 2 \\
\hline Normalidade & 2 & Miocardite linfocitária & 2 \\
Infarto antigo & 2 & Normalidade & 1 \\
Miocardite & 1 & Cardiopatia hipertrófica & 5 \\
Total & 5 & Total & 5 \\
\hline
\end{tabular}




\section{Tabela 3 Diagnósticos microscópicos que não foram vistos na macroscopia}

\begin{tabular}{|c|c|c|c|c|c|c|c|}
\hline \multicolumn{2}{|l|}{ Pulmão } & \multicolumn{2}{|l|}{ Rim } & \multicolumn{2}{|l|}{ Fígado } & \multicolumn{2}{|c|}{ Coração } \\
\hline Broncopneumonia & 12 & Pielonefrite crônica & 19 & Esteatose & 12 & Infarto antigo & 9 \\
\hline Enfisema & 10 & Nefrosclerose & 14 & Hepatite aguda & 7 & Cardite aguda & 4 \\
\hline Hemorragia & 9 & Necrose tubular aguda & 8 & Hepatite crônica & 4 & IAM & 4 \\
\hline Edema & 8 & Adenoma & 2 & Cirrose & 3 & Chagas & 2 \\
\hline DPOC & 7 & $\begin{array}{l}\text { Degeneração } \\
\text { hidrópica }\end{array}$ & 2 & Reação portal & 2 & Hipertrofia & 2 \\
\hline Hipertensão & 5 & Infarto & 1 & Congestão & 2 & Esclerose & 2 \\
\hline $\begin{array}{l}\text { Dano alveolar } \\
\text { difuso }\end{array}$ & 4 & Outros & 3 & Outros & 7 & Outros & 3 \\
\hline Congestão & 3 & - & - & - & - & - & - \\
\hline Infarto & 3 & - & - & - & - & - & - \\
\hline Outros & 10 & - & - & - & - & - & - \\
\hline Total & 71 & Total & 49 & Total & 37 & Total & 26 \\
\hline
\end{tabular}

\section{Discussão e conclusão}

Em nosso estudo, as causas básicas de óbito mais freqüentes foram as doenças cardiovasculares, e as terminais foram as doenças respiratórias. O pulmão foi o órgão com maior discrepância diagnóstica, o mesmo achado referido

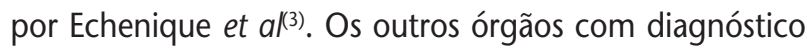
divergente foram os rins, o fígado e o coração. Ao contrário do estudo de Echenique, nós achamos melhor não dividir os erros em leves e graves, pois o trabalho foi retrospectivo, com dados provenientes de diversos patologistas, sem que houvesse discussão dos diagnósticos pela equipe. A discordância entre os diagnósticos de causa básica de óbito foi de $22 \%$ e, em relação à causa imediata, de $16 \%$. Uma das preocupações sobre a dificuldade no diagnóstico macroscópico é em relação ao uso dos dados do AO como ferramenta de saúde pública. Um erro em relação à causa básica de um $\mathrm{AO}$ pode gerar grandes mudanças em termos epidemiológicos, como, por exemplo, mudar de um problema infeccioso para uma neoplasia. Como a análise dos atestados se dá em relação ao Código Internacional de Doenças (CID), resolvemos comparar os erros baseados também nessa classificação. Em relação à causa básica, mesmo nos 22 casos discordantes, $86 \%$ mantiveram a mesma classificação do CID; enquanto que, em relação à causa imediata, dos 16 casos discordantes, 89\% não mudaram de CID. Em ambas as causas, imediatas ou básicas, as diferenças no CID ocorreram principalmente em relação às doenças cardiovasculares e respiratórias.

Existem diversos motivos para a ocorrência de erros diagnósticos. Uns são inerentes à limitação do exame macroscópico, como, por exemplo, o diagnóstico etiológico de uma hepatopatia e outros dependem da experiência do patologista e das informações clínicas que o mesmo tem à disposição no momento da autópsia. Além desses, muitas doenças se sobrepõem macroscopicamente. No pulmão, por exemplo, broncopneumonia, síndrome da angústia respiratória aguda (SARA) e, às vezes, hemorragia pulmonar podem ser bastante semelhantes. Isso sem contar o diagnóstico etiológico da lesão, como broncopneumonia e tuberculose, fato também observado por Echenique et al. ${ }^{(3)}$. No nosso trabalho observamos, ainda em relação aos pulmões, pouco diagnóstico macroscópico de enfisema, apenas microscópico (Tabela 4). Provavelmente isso se deve ao fato de não se dar muita importância a esse diagnóstico, considerando-o secundário e sendo descrito somente no relatório final. O mesmo fato se observa em outras doenças, como pielonefrite crônica e infarto antigo do miocárdio.

Por ser um estudo retrospectivo, nosso trabalho apresenta muitas críticas metodológicas. Além da variação de patologistas (seis no total), com diferentes formações e especialidades, não existe padronização em relação ao número de cortes histológicos de cada órgão, não havendo 


\section{Tabela 4 Diagnósticos microscópicos divergentes e seus respectivos achados macroscópicos}

\section{a) Pulmão}

\begin{tabular}{l|c|l|l|l|l|l|c}
\hline \multicolumn{2}{l|}{ Broncopneumonia (micro) } & Enfisema (micro) & Hemorragia (micro) & \multicolumn{2}{l}{ Edema (micro) } \\
\hline Normalidade & 6 & Normalidade & 6 & Edema & 3 & Normalidade & 5 \\
Edema & 2 & Broncopneumonia & 2 & Broncopneumonia & 2 & Congestão & 2 \\
Hemorragia & 2 & Edema & 2 & Congestão & 2 & Broncopneumonia & 1 \\
SARA & 1 & - & - & Normalidade & 1 & - & - \\
Granulomatose & 1 & - & - & Tromboembolismo & 1 & - & - \\
Total & 12 & Total & 10 & Total & 9 & Total & $\mathbf{8}$ \\
\hline
\end{tabular}

b) $\operatorname{Rim}$

\begin{tabular}{l|c|l|c|l|c}
\hline Pielonefrite (micro) & & Nefrosclerose (micro) & \multicolumn{2}{|l}{ Necrose tubular aguda (micro) } \\
\hline Normalidade & 14 & Normalidade & 12 & Normalidade & 8 \\
Nefroesclerose & 4 & Necrose tubular aguda & 2 & - & - \\
Necrose tubular aguda & 1 & - & - & - & - \\
Total & 19 & Total & 14 & Total & 8 \\
\hline
\end{tabular}

\section{c) Fígado}

\begin{tabular}{l|c|l|c|l|r}
\hline \multicolumn{2}{l|}{ Esteatose (micro) } & Hepatite aguda (micro) & \multicolumn{2}{l}{ Hepatite crônica (micro) } \\
\hline Normalidade & 7 & Normalidade & 4 & Hepatite aguda & 2 \\
Hepatite aguda & 2 & Esteatose & 2 & Esteatose & 1 \\
Congestão & 2 & Hepatite crônica & 1 & Normalidade & 1 \\
Hepatomegalia & 1 & - & - & - & - \\
Total & 12 & Total & $\mathbf{7}$ & Total & $\mathbf{4}$ \\
\hline
\end{tabular}

d) Coração

\begin{tabular}{l|r|l|c}
\hline \multicolumn{2}{l|}{ Infarto antigo (micro) } & \multicolumn{2}{l}{ IAM (micro) } \\
\hline IAM & 4 & Normalidade & 3 \\
Normalidade & 3 & Infarto antigo & 1 \\
Hipertrofia miocárdica & 2 & - & - \\
Total & $\mathbf{9}$ & Total & 4 \\
\hline
\end{tabular}

SARA = síndrome da angústia respiratória do adulto.

uma padronização por parte dos patologistas. Enquanto que alguns faziam muitos cortes histológicos de pulmão, por exemplo, outros faziam apenas dois. Não foi infreqüente, segundo observamos, que o patologista e o residente tivessem que fazer mais cortes dos órgãos quando havia discrepância nos diagnósticos macro e microscópico. Por exemplo, havia casos em que a macroscopia observava uma broncopneumonia e, na micro, isso não era observado num primeiro momento. Só depois, com mais cortes histológicos, encontrava-se a lesão. Essas evidências nos mostram claramente a importância do estudo microscópico, de maneira relevante quando há um certo grau de subjetividade ao analisar a macroscopia, principalmente quando estudamos pulmões, fígado, rins e coração. No entanto, esse estudo deve ser realizado de maneira racional, com protocolos estabelecidos na dependência da rotina do serviço de patologia local. Para que nenhuma afecção deixe de ser diagnosticada microscopicamente, é preciso estabelecer um número razoável de cortes de fragmentos direcionados aos órgãos principais por autópsia, sem, contudo, executar a confecção de lâminas de maneira desenfreada, onerando os gastos com saúde. 
Resgatando o título do trabalho, referente à análise das dificuldades e às possíveis causas de erro e discordâncias macro e microscópica, gostaríamos de ressaltar algumas evidências que freqüentemente ocorrem nos serviços de patologia em grandes centros, principalmente nos que estão vinculados a um hospital universitário com residência de patologia: 1) os diagnósticos macroscópicos das afecções do trato digestório dificilmente são confirmados na microscopia, em função da autólise do material; 2) muitas informações são desprezadas na elaboração do laudo definitivo, pois são muito comuns e não são interpretadas como importantes no raciocínio diagnóstico (por exemplo, enfisema pulmonar focal, aterosclerose, etc.); 3) o grande número de patologistas, com formação e experiência diferentes, que realizam as necropsias e que podem levar a um viés do diagnóstico (por exemplo, um patologista pulmonar vai prestar mais atenção nos pulmões do que no fígado e vice-versa); 4) infelizmente existem ainda muitos erros no preenchimento do AO por desconhecimento das normas de preenchimento (baseados na macroscopia), emitindo-se documentos incompletos. Além disso, muitas vezes, o laudo provisório é modificado após o estudo dos fragmentos histopatológicos. Assim, o AO, embora muito utilizado, apresenta uma limitação como ferramenta epidemiológica, pois o diagnóstico não é o definitivo.

\section{Agradecimentos}

À FAPESP, pelo auxílio de bolsa de iniciação científica, processo 00/06625-2.

\section{Referências}

I. BLOSSER, A. S.; ZIMMERMAN, H. E.; STAUFFER, J. L. Do autopsies of critically ill patients reveal important findings that were clinically undetected? Crit Care Med, v. 26, p. 1332-6, 1998.

2. CARVALHO, F. M. et al. Clinical diagnosis versus autopsy. Bull Pan Am Health Organ, v. 25, p. 4I-6, I991.

3. ECHENIQUE, L. S. et al. Correlação entre achados macro e microscópico em 200 autópsias consecutivas: análise do valor custo/benefício do estudo histopatológico completo das autópsias. Jornal Brasileiro de Patologia, v. 38 p. I19-224, 2002.
4. JURIC, G.; TENTOR, D.; JAKIC-RAZUMOVIC, J. Autopsy findings and clinical diagnoses: retrospective study of 3,117 autopsies. Croat Med J, v. 40, p. 7I-6, 1999.

5. MOSQUERA, D. A.; GOLDMAN, M. D. Surgical audit without autopsy: tales of the unexpected. Am R Coll Surg Engl, v. 175, p. I15-7, 1993

6. VERESS, B. et al. The reliability of autopsiy diagnostics: inter-observer variation between pathogists, a preliminary report. Qual Assur Health Care, v. 5, p. 333-7. 1993.
Endereço para correspondência

Patricia M. Cury

Departamento de Patologia da FAMERP Av. Brigadeiro Faria Lima 5.416

CEP 15090-000 - São José do Rio Preto-SP

e-mail: pmcury@famerp.br 